\title{
Immunogenic properties of recombinant ectodomain of Newcastle disease virus hemagglutinin-neuraminidase protein expressed in Escherichia coli.
}

\begin{abstract}
Hemagglutinin-neuraminidase (HN) protein of Newcastle disease virus (NDV) plays a vital role in the viral infectivity, host immunity, and disease diagnosis. A portion of the HN gene encoding the ectodomain (nt 142-1739) was cloned and expressed in Escherichia coli yielding an insoluble $\mathrm{HN}$ protein and a soluble NusA-HN protein containing N-utilization substance A (NusA) fusion component. Both recombinant proteins were purified and used for immunization of chickens. The recombinant HN protein induced higher antibody titers as compared to the recombinant NusA-HN protein. These antibodies were able to react in immunoblot analysis with the corresponding recombinant proteins as well as with the $\mathrm{HN}$ protein of NDV.
\end{abstract}

Keyword: Newcastle disease virus; Hemagglutinin-neuraminidase; Cloning; Expression; Immunogenicity. 